\title{
Cognition in mood disorders
}

Katie M. Douglas, Richard J. Porter and Allan H. Young

Cognitive impairment plays a key role in determining the course of illness and functional outcomes in mood disorders. This article summarises and discusses important papers within this thematic series of BJPSych Open that contribute to a greater understanding of the complexity of 'Cognition in Mood Disorders'.

\section{Keywords}

Bipolar affective disorders; depressive disorders; cognition; neuropsychology; mood disorders.

\section{Copyright and usage}

(c) The Authors 2020. Published by Cambridge University Press on behalf of the Royal College of Psychiatrists. This is an Open Access article, distributed under the terms of the Creative Commons Attribution licence (http://creativecommons.org/ licenses/by/4.0/), which permits unrestricted re-use, distribution, and reproduction in any medium, provided the original work is properly cited.
Dr Katie Douglas is a senior research fellow/Sir Charles Hercus Fellow, and Clinical Psychologist at the Department of Psychological Medicine at University of Otago, New Zealand Professor Richard Porter is a Consultant Psychiatrist and Head of the Department of Psychological Medicine at University of Otago, New Zealand. Professor Young is Chair of Mood Disorders at the Institute of Psychiatry, Psychology \& Neuroscience. His research interests focus on the cause and treatments for severe psychiatric illnesses, particularly mood disorders.

Aspects of cognitive functioning play a vital role in determining the course of illness and functional outcome for individuals with mood disorders. Broadly speaking, cognitive functioning can be divided into emotional processing, or what has been termed 'hot' cognition, and non-emotional or 'cold' cognition. The distinction is not absolute, but in general it appears that abnormalities or biases in emotional processing may be more closely related to current mood state in mood disorders, and it is suggested that normalisation of these biases may be important for response to treatment. Cold cognition may have some relationship to mood state, particularly when this is severely perturbed, but abnormalities in these aspects of cognitive function have been found to persist into remission or euthymia. This thematic series of BJPsych Open addresses both aspects of cognition.

\section{Hot cognition}

Significant evidence has accumulated regarding abnormalities in emotional processing in depression. Less clear evidence has been produced in bipolar disorder, although abnormalities in facial expression recognition and implicit emotion regulation have been more consistently found. ${ }^{1}$ Studies in depression have led to the cognitive neuropsychological hypothesis, ${ }^{2}$ which posits that negative biases in emotional processing are an important part of the pathophysiology of depression, and that successful treatments for depression reverse these, allowing more rewarding interactions and providing a positive feedback loop that continues the process of recovery from an episode of depression. According to this theory, potential treatments for depression could be screened based on whether they reverse emotional processing deficits, and if they do not, then they are unlikely to be effective antidepressants. Furthermore, the hypothesis that changes in emotional processing are a first step toward more positive social interactions and full recovery has begun to be investigated. Hobbs et $\mathrm{al}^{3}$ (this series) present data on the effects of citalopram in healthy volunteers in a randomised between-group study. In computerised paradigms evaluating referential affective processing, social cognition and expression of prosocial behaviour, participants who received a single dose of citalopram showed greater prosocial behaviours toward others. Exploratory analyses also suggested increased positive affective biases and increased positive recall after administration of citalopram, suggesting, as per the cognitive neuropsychological hypothesis, that there is a shift with this antidepressant toward a more positive appraisal of the environment.

Much of the research on emotional processing biases in depression and anxiety disorders has been conducted in younger people. However, with an ageing population, it is important to consider older adults, particularly given well-established data on changes in emotional processing with ageing. Gray et $\mathrm{al}^{4}$ (this series) examine evidence for changes in emotional processing in late-life depression and anxiety disorders. This is reviewed in light of the 'positivity bias' (the tendency of older adults to attend to, and remember, positive information more than negative information) in emotion processing that is observed in older adults. ${ }^{5}$ This might be expected to have a differential effect on emotional processing in late-life depression and anxiety compared with depression and anxiety in younger people. The review found relatively little evidence of impaired emotional processing in late-life depression, although changes in brain circuitry in this group were found to be broadly similar to those seen in younger patients. Evidence was more consistent in late-life anxiety and post-traumatic stress disorder, where there was clearer evidence of a bias toward attending to negative or threat-related stimuli, as in younger people.

\section{Cold cognition}

Although meta-analyses have consistently suggested that impairment in cold cognition persists into euthymia, ${ }^{6,7}$ more recent research has focused on understanding the very significant heterogeneity in the degree of cognitive impairment seen in mood disorders. ${ }^{8}$ In attempting to understand this, several studies have used cluster analytic techniques to generate groups with different cognitive profiles. These have generally characterised three or four profiles, of which one has been a group of patients with relatively normal cognitive function compared with population norms or healthy controls, whereas one group has generally been characterised by global cognitive impairment. In Tsapekos et $\mathrm{al}^{9}$ (this series), to attempt to understand the predictors of these cognitive profiles, four separate profiles were identified in patients with bipolar disorder. Interestingly, but perhaps not surprisingly, lower cognitive reserve was associated with membership of higher 
impairment groups. The only other factor identified was the use of antipsychotics, which was significantly lower in the intact group.

In an attempt to understand what predicts improvement in cognitive function in the treatment of major depressive episodes, Barczyk et $\mathrm{al}^{10}$ (this series) examined studies that measured cognitive function at baseline and at follow-up, after treatment of an episode of major depression. Once again, age was a significant issue in the review, with studies being best understood by a split into younger and older patients. There was some evidence that increased severity of depression at baseline and increased severity of cognitive impairment at baseline both predicted a greater degree of cognitive change with treatment. These variables may be features that could be used in selecting suitable patients for future trials of, for example, cognitive remediation.

\section{Subjective versus objective impairment of cognitive function}

It is increasingly recognised that people's subjective view of their cognitive ability may have considerable importance, even if this does not accord with results of objective cognitive testing. Research has focused on factors that make people more likely to experience subjective compared with objective impairment. ${ }^{11}$ For example, expectations may be important in determining subjective assessment of cognitive function. An interesting recent report in BJPsych Open ${ }^{12}$ suggested that patients who held a negative expectation of the effect that electroconvulsive therapy would have on their memory were more likely to have subjective memory worsening. Less research about subjective cognitive function has been conducted in youth depression samples, whose experience of cognitive problems associated with depression may be different if depression onset is relatively recent. In their analysis of depression symptoms over 3 months, and perceived change in subjective cognitive function, Allott et $\mathrm{al}^{13}$ (this series) reported that improvement or reduction in self-rated depression symptoms was highly associated with change in subjective ratings of cognitive function in their youth sample. That is, patients who reported improvement in their mood also felt their cognitive functions had strengthened. This was the case even though severity of depression in this young sample was moderate. A further interesting finding was that those with greater severity of depression at baseline were more likely to report a decline in subjective cognitive functioning over the subsequent 3 months. Much research to date has focused on objective cognitive functioning, but subjective cognitive functioning may be equally important in determining outcomes for patients. ${ }^{14}$ Therefore, development of interventions aimed at improving patients' perception of cognitive functions may be helpful in enhancing recovery from mood disorders.

\section{Treatment of cognitive impairment}

To date, there is relatively little evidence for treatments that might improve cognitive function in the group of patients for whom this is impaired long-term. ${ }^{15}$ However, negative trials may be explained to an extent by methodological issues. Guidelines have been written regarding appropriate methodology for cognitive enhancement trials in bipolar disorder, ${ }^{16}$ which have emphasised the need to screen for significant cognitive impairment and to wait until euthymia is achieved before commencing cognitive remediation strategies. Some recent studies, however, are attempting to treat cognitive impairment even when patients are still acutely depressed, with cognitive exercises hypothesised to be working by activating prefrontal brain areas. ${ }^{17}$ Cognitive remediation may also be useful in patients who are chronically depressed and have ongoing cognitive impairment. The field is relatively new, and it is therefore timely to produce recommendations and a review of methodology of cognitive enhancement studies in major depressive disorder. Douglas et $\mathrm{al}^{18}$ (this series) do this, not only examining methodologies for trials, but also attempting to give some guidance for clinicians who may wish to provide cognitive interventions for patients who are particularly impaired.

\section{Relationship of cognitive impairment associated with mood disorders and onset of dementia}

In late-life depression and bipolar disorder, understanding cognitive function is complicated by the relationship between the mood disorders and dementia, with episodes of mood disorder being associated with risk of dementia. ${ }^{19}$ Barczyk et al ${ }^{10}$ (this series), in their review of factors that predict cognitive change, note that in some longer-term studies in late-life depression, cognitive change is predominantly negative. These studies examine what predicts a decline, finding that, for example, the number of deep white matter lesions appears to be a significant factor. A factor whose association with dementia is well established is apolipoprotein E $\varepsilon 4\left(\mathrm{APOE}^{*} \varepsilon 4\right)$ allele. The relationship between $\mathrm{APOE}^{\star} \varepsilon 4$ and depression is less clear. Burns et $\mathrm{al}^{20}$ (this series) showed convincing longitudinal evidence across the adult lifespan that in cognitively intact, non-depressed adults at baseline, $\mathrm{APOE}^{\star} \varepsilon 4$ was not associated with incident depression risk. As with in younger adult samples, further research examining cognitive trajectories in old-age depression samples would be beneficial in determining whether presence of certain factors (e.g. $\mathrm{APOE}^{*} \varepsilon 4$ or deep white matter lesions) would affect cognitive remediation strategies.

\section{Conclusions}

In summary, research examining the issue of cognitive impairment in mood disorders has revealed increasing complexity in recent years. Distinctions include between hot and cold cognition, between objective and subjective cognitive impairment, and between cognitive impairment associated with euthymia and that associated with the episodes of illness. It has become apparent that the degree of cognitive impairment is variable between individuals, with the beginnings of evidence regarding what predicts who will suffer from significant impairment. Studies have begun to examine techniques to treat cognitive impairment, but the methodological issues are complex. As patients age, this affects the profile and likely outcome of cognitive impairment associated with mood disorders. This thematic series of BJPsych Open touches on all these subtleties, as well as suggesting future research directions, particularly in the area of cognitive remediation.

Katie M. Douglas (D), Department of Psychological Medicine, University of Otago, New Zealand; Richard J. Porter (D), Specialist Mental Health Services, Canterbury District Health Board, New Zealand; and Department of Psychological Medicine, University of Otago, New Zealand; Allan H. Young (D), Department of Psychological Medicine, Institute of Psychiatry, Psychology and Neuroscience, King's College London, UK; and south London and Maudsley NHS Foundation Trust, Bethlem Royal Hospital, UK

Correspondence: Dr Katie Douglas. Email: katie.douglas@otago.ac.nz

First received 21 Oct 2020, final revision 16 Nov 2020, accepted 16 Nov 2020

\section{Author contributions}

K.M.D. and R.J.P. jointly prepared this article and edited subsequent revisions. A.H.Y. provided comments for the final draft. All authors were involved in final approval of the paper. 


\section{Funding}

K.M.D. was supported by a Sir Charles Hercus Health Research Fellowship from the Health Research Council of New Zealand during completion of this article. A.H.Y.'s research is funded by the National Institute for Health Research (NIHR) Biomedical Research Centre at South London and Maudsley NHS Foundation Trust and King's College London. The views expressed are those of the authors and not necessarily those of the NHS, the NIHR or the Department of Health.

\section{Declaration of interest}

K.M.D. and RJ.P. use software provided free-of-charge by Scientific Brain Training Pro for cognitive remediation trials. R.J.P has received support for travel to educational meetings from servier and Lundbeck. A.H.Y. has received funding and sat on advisory boards for AstraZeneca, Eli Lilly, Lundbeck, Sunovion, Servier, LivaNova, Janssen, Allegan, Bionomics, Sumitomo Dainippon Pharma and COMPASS. He is a consultant to Johnson \& Johnson and Livanova.

\section{References}

1 Miskowiak KW, Seeberg I, Kjaerstad HL, Burdick KE, Martinez-Aran A, Del Mar Bonnin C, et al. Affective cognition in bipolar disorder: a systematic review by the ISBD targeting cognition task force. Bipolar Disord 2019; 21: 686-719.

2 Godlewska BR, Harmer CJ. Cognitive neuropsychological theory of antidepressant action: a modern-day approach to depression and its treatment. Psychopharmacology (Berl) [Epub ahead of print] 15 Jan 2020. Available from: https://doi.org/10.1007/s00213-019-05448-0.

3 Hobbs C, Murphy SE, Wright L, Carson J, Assche IV, O'Brien J, et al. Effect of acute citalopram on self-referential emotional processing and social cognition in healthy volunteers. BJPsych Open 2020; 6: e124.

4 Gray V, Douglas KM, Porter RJ. Emotion processing in depression and anxiety disorders in older adults: systematic review. BJPsych Open, 2020, in press.

5 Carstensen LL, DeLiema M. The positivity effect: a negativity bias in youth fades with age. Curr Opin Behav Sci 2018; 19: 7-12

6 Robinson L, Thompson JM, Gallagher P, Goswami U, Young AH, Ferrier IN, et al. A meta-analysis of cognitive deficits in euthymic patients with bipolar disorder. J Affect Disord 2006; 93: 105-15.

7 Mann-Wrobel MC, Carreno JT, Dickinson D. Meta-analysis of neuropsychological functioning in euthymic bipolar disorder: an update and investigation of moderator variables. Bipolar Disord 2011; 13: 334-42.

8 Burdick KE, Russo M, Frangou S, Mahon K, Braga RJ, Shanahan M, et al. Empirical evidence for discrete neurocognitive subgroups in bipolar disorder: clinical implications. Psychol Med 2014; 44: 3083-96.
9 Tsapekos D, Strawbridge R, Mantingh T, Cella M, Wykes T, Young AH. Role of cognitive reserve in cognitive variability in euthymic individuals with bipolar disorder: cross-sectional cluster analysis. BJPsych Open 2020; 6: e133.

10 Barczyk ZA, Douglas KM, Porter RJ. Baseline predictors of cognitive change in the treatment of major depressive episode: systematic review. BJPsych Open 2020; 6: e131.

11 Petersen JZ, Porter RJ, Miskowiak KW. Clinical characteristics associated with the discrepancy between subjective and objective cognitive impairment in depression. J Affect Disord 2019; 246: 763-74.

12 Sigstrom R, Nordenskjold A, Jureus A, Clements C, Joas E, Palsson E, et al. Longterm subjective memory after electroconvulsive therapy. BJPsych Open 2020; 6: e26.

13 Allott K, Gao C, Hetrick SE, Filia KM, Menssink JM, Fisher C, et al. Subjective cognitive functioning in relation to changes in levels of depression and anxiety in youth over 3 months of treatment. BJPsych Open 2020; 6: e84.

14 Saragoussi D, Touya M, Haro JM, Jonsson B, Knapp M, Botrel B, et al. Factors associated with failure to achieve remission and with relapse after remission in patients with major depressive disorder in the PERFORM study. Neuropsychiatr Dis Treat 2017; 13: 2151-65

15 Douglas KM, Van Rheenen TE. Current treatment options for cognitive impairment in bipolar disorder: a review. Curr Treat Options Psychiatry 2016; 3: $330-55$

16 Miskowiak KW, Burdick KE, Martinez-Aran A, Bonnin CM, Bowie CR, Carvalho $A F$, et al. Methodological recommendations for cognition trials in bipolar disorder by the International Society for Bipolar Disorders Targeting Cognition Task Force. Bipolar Disord 2017; 19: 614-26.

17 Averill IRE, Beaglehole B, Douglas KM, Jordan J, Crowe MT, Inder M, et al. Activation therapy for the treatment of inpatients with depression - protocol for a randomised control trial compared to treatment as usual. BMC Psychiatry 2019; 19: 52

18 Douglas KM, Milanovic M, Porter RJ, Bowie CR. Clinical and methodological considerations for psychological treatment of cognitive impairment in major depressive disorder. BJPSych Open 2020; 6: e67.

19 Kessing LV Andersen PK. Does the risk of developing dementia increase with the number of episodes in patients with depressive disorder and in patients with bipolar disorder? J Neurol Neurosurg Psychiatry 2004; 75: 1662-6.

20 Burns RA, Andrews S, Cherbuin N, Anstey KJ. Role of apolipoprotein E epsilon 4 (APOE*epsilon4) as an independent risk factor for incident depression over a 12-year period in cognitively intact adults across the lifespan. BJPsych Open 2020; 6: e47. 